Recebido em 11/2019. Aceito para publicação em 01/2020.

\title{
TIJOLOS COMPÓSITOS DO TIPO SANDUÍCHE DE CONCRETO-POLÍMERO-METAL
}

\section{COMPOSITE BRICKS OF THE TYPE CONCRETE-POLYMER-METAL SANDWICH}

\author{
Fabiana Teodosio Barbosa ${ }^{1}$ \\ Alex da Silva Sirqueira ${ }^{2}$
}

\begin{abstract}
Resumo: O poliestireno expandido (EPS) é um material que vem sendo difundido na indústria da construção, com vistas à sustentabilidade e preservação ambiental, pois o EPS é $100 \%$ reciclável. Nesse contexto, essa pesquisa objetivou estudar as propriedades mecânicas e físicas deste material associado com concreto e metal. Os ensaios testados formam a resistência à compressão, absorção de água, dureza e o processo de corrosão. Buscou-se produzir, um tijolo com três componentes: um bloco de polímero tipo 4 (alocado no centro), malha metálica (para revesti-lo) e argamassa. Através dos ensaios, foi observado que os tijolos convencionais absorvem mais água quando comparados ao compósito desenvolvido. Os CPs submetidos ao ensaio de exposição à temperatura de $50^{\circ} \mathrm{C}$ perderam massa específica, mas os resultados demostram que não houve perda da resistência à compressão. A deformação manteve-se dentro dos padrões da norma. Ao analisar a força de compressão conclui-se que mesmo o compósito com menor resistência, suportou carga maior que 8,1 ff/ $\mathrm{m}^{2}$ do EPS. Nesse contexto, pode-se deduzir que o aço e a argamassa oferecem maior resistência ao material e que, assim sendo, compósitos estudados são capazes de suportar as cargas quando estão distribuídas por $m^{2}$. Os materiais submetidos ao ensaio de corrosão apresentaram valores dentro da norma.
\end{abstract}

Palavras-chave: Compósitos; propriedades mecânicas.

Abstract: EPS (expanded polystyrene) is a material that is widespread in the construction industry for sustainability, since EPS is $100 \%$ recyclable. In this context, this research aimed to study the mechanical and physical properties of this material associated with concrete and metal. The tests analyzed the compressive strength, water absorption, hardness and corrosion process. In this work, we tried to produce a brick with three components: a type 4 polymer block (centrally allocated), metallic mesh (to cover it) and mortar, always focusing on the synergism of the properties. 18 (eighteen) CPS and two brick samples were used. By the tests, it was observed that the conventional bricks absorb more water compared to the developed composite. The CPs submitted to the temperature of $50^{\circ} \mathrm{C}$ lost specific mass, but the results show that there was no loss of compressive strength, and the deformation remained inside the normative standards, when compared to the results of other CPs that did not undergo the process. Analyzing the strength found, it can be concluded that even the composite that presented the lowest resistance supported a load higher than $8.1 \mathrm{tf} / \mathrm{m}^{2}$ of EPS. In this context, it can be deduced that steel and mortar offer greater resistance to the material and, therefore, the studied composites are able to withstand the loads when they are distributed per $\mathrm{m}^{2}$. However, when the composites were analyzed as simple compression masonry (wall), they would only meet those that had a resistance greater than $92.55 \mathrm{kN}$ and a deformation of $86 \%$, because they presented a resistance of $203.43 \mathrm{kN} / \mathrm{m}^{2}$.

Keywords: Composites; mechanical properties.

\section{INTRODUÇÂO}

Uma alternativa para minimizar o consumo de materiais não recicláveis na

\footnotetext{
1 Graduada, Engenheira Civil. Fundação Centro Universitário Estadual da Zona Oeste, UEZO, Brasil. E-mail: biana2705@gmail.com.

2 Doutor, Professor da Fundação Centro Universitário Estadual, da Zona Oeste, UEZO, Brasil. E-mail: assirqueira@gmail.com.
} 
construção civil é o uso do poliestireno expandido (EPS) para construções estruturais. O EPS é um material polimérico 100\% reciclável, de baixa densidade, resistente a intemperes e ao envelhecimento. Ademais, pode-se ressaltar que os sistemas construtivos com EPS são formas úteis para a construção civil, pois apresentam maior agilidade e redução no custo da obra, gerando, ainda, benefícios de conforto térmico e acústico. Por sua vez, a aplicação desse material não compromete a segurança da construção, chegando a reduzir em até $20 \%$ o custo de fundação da obra (SANTOS et al., 2009).

Assim, é de grande avalia aplicar EPS como peça estrutural, pois é um material que pesa entre 13 a $25 \mathrm{~kg} / \mathrm{m} 3$, e tem resistência à compressão de 1 a $2 \mathrm{~kg} / \mathrm{cm} 2$. No mercado, os tipos de EPS são encontrados em escala de 1 a 7 , sendo que quanto maior for o tipo, maior é a sua densidade e, logo, apresentará melhor resistência à compressão (ASSOCIAÇÃO BRASILEIRA DE POLIESTIRENO EXPANDIDO, 2010).

O poliestireno expandido tem diversas aplicações na construção civil e, segundo Lopes (2011), este material é adotado para isolamento de paredes tanto da parte interior, quanto da parte exterior de edificações.

Atualmente, existe no mercado um novo tipo de telha termoacústica, cujo núcleo é composto por poliestireno expandido (EPS). Pelo fato desta telha conter material isolante em seu miolo, haverá menor consumo de energia na fase operacional da habitação (CALDEIRA; SPOSTO; CALDAS, 2016).

Embora, o EPS apresente inúmeras vantagens em relação ao tijolo de concreto, não se pode simplesmente fazer uma parede somente com este material, pois tornaria a construção vulnerável.

Segundo Stocco, Rodrigues e Castro (2009), o concreto com EPS é aplicado em diversos tipos de construções tais como:

Pré-fabricados:

a) Elementos de vedação internos (paredes);

b) Isolante acústico;

c) Isolante térmico;

d) Isolante térmico e acústico de lajes;

e) Resistência à propagação do fogo;

f) Muros exteriores sem carga;

g) Casas pré-fabricadas;

h) Tijolos ou blocos de concreto leve;

i) Revestimento de fachadas com concreto leve;

j) Elementos vazados de concreto leve.

Globalmente, a construção de edifícios é responsável pelo uso de $40 \%$ dos 
recursos minerais (pedra, brita, areia, etc.), por $25 \%$ da madeira, $40 \%$ da energia e $16 \%$ da água consumidos anualmente (MATEUS; BRAGANÇA, 2004). Logo, a busca do desenvolvimento sustentável tem sido tema de diversas pesquisas e estudos e, assim, a necessidade de uma arquitetura e construção sustentáveis tem fomentado a investigação de produtos alternativos. Portanto, a aplicação do EPS é uma prática de sustentabilidade, já que a geração de resíduos é baixa e os gerados são reaproveitados, pois possuem potencial de aplicabilidade (SANTOS, 2009).

Na Europa, a construção civil responde por $65 \%$ do consumo de EPS, enquanto que no Brasil esse percentual chega a apenas $4 \%$. Supõe-se, entretanto, que isso se dá pela dificuldade em fazer o resíduo de EPS sair da residência do consumidor e voltar à indústria transformadora (SANTOS, 2019). Ainda, é premente salientar que a reciclagem do poliestireno elimina as várias etapas iniciais da fabricação da matéria prima virgem, diminuindo gastos com energia e a emissão de poluentes relacionados ao processo de obtenção do poliestireno expandido (SANTOS, 2013).

Os materiais utilizados na construção civil ocasionam grande impacto ambiental. Logo, torna-se fundamental que, dentro de uma obra, seja selecionado um material que cumpra a função requerida com o mínimo impacto ambiental e que, simultaneamente, garanta o necessário desempenho técnico adequado e seja viável economicamente (JOHN; SATO, 2006).

Nesse contexto, pode-se afirmar que a aplicação do EPS na construção civil é de grande vantagem, pois permite a redução de uso de cimento, já que este ocasiona grandes impactos ambientais, uma vez que se faz necessário uma grande aplicação de energia no processo de calcinação do cimento; ademais, a sua fabricação é responsável por um vultoso volume da emissão de CO2. Segundo John, Sjöström e Agopyan (2002), a indústria cimenteira brasileira é responsável por mais de $10 \%$ do $\mathrm{CO} 2$ emitido no Brasil.

Mesmo sabendo que o calcário é importante matéria-prima para a produção de vários materiais de construção (em especial a do cimento), deve-se adotar novas tecnologias para diminuir a utilização deste, pois, a cada tonelada de calcário calcinado, são liberados $440 \mathrm{~kg}$ de CO2 para a atmosfera. Logo, esse processo contribui para o aquecimento global e para outros impactos ambientais importantes e que dependem dos combustíveis e de processos, como chuvas ácidas, emissão de dioxinas etc. (JOHN; SATO, 2006).

Diante das circunstâncias mencionadas anteriormente, pode-se mencionar que o uso de concreto convencional pode ser substituído por uma nova tecnologia adotada para construção de edificações: os painéis monolíticos de EPS. Essa tecnologia consiste em um painel de EPS envolvido com duas malhas de arame de aço eletrosoldadas que, quando projetada na argamassa (emboço), diminuirá o consumo de cimento. A Figura 2 permite a percepção da projeção de argamassa. 
Portanto, a pesquisa objetiva-se em princípio, estudar as propriedades mecânicas e físicas do compósito tipo sanduiche de EPS-malha metálica-argamassa.

Neste contexto, buscou-se produzir, no trabalho, um tijolo com os três componentes, cuja composição se caracterizou por um bloco de polímero alocado no centro, sendo revestido pela malha metálica e argamassa. O foco do estudo, é o sinergismo de propriedades.

\section{MATERIAIS E MÉTODOS}

\subsection{Materiais}

Poliestireno expandido (EPS) tipo 4, material retardante de chama com densidade aparente nominal 18 , densidade aparente mínima 16 , condutividade térmica máxima $\left(23^{\circ} \mathrm{C}\right) 0,039$, tensão por compreensão com deformação de $10 \% \geq 80$, resistência mínima à flexão $\geq 160$ e resistência mínima ao cisalhamento $\geq 80$, fabricante Knauf, dona da marca Isoporß.

Para envolver o EPS, foi utilizada a tela de aço média de $3,4 \mathrm{~mm}$, malha de $15 \times 15 \mathrm{~cm}$ e painel de $2 \times 3 \mathrm{~m}$. A tela é feita com vergalhões de aço trançados e amarrados com arames, formando uma espécie de tela.

Para o embolso do EPS foi usado um traço de 3:2: 1 (areia, aréola e cimento).

\subsection{Preparação do compósito}

Para preparação dos compósitos, foram usados blocos de EPS tipo 4 (quatro) com as seguintes dimensões: $19 \mathrm{~cm} \times 19 \mathrm{~cm} \times 9 \mathrm{~cm}$ e $29 \mathrm{~cm} \times 19 \mathrm{~cm} \times 9$. Estes foram envolvidos com uma tela de aço, as quais foram cortadas em dimensões menores $\mathrm{e}$ presas com arrame queimado.

Após a fixação da tela do EPS, foi realizada a preparação do traço de massa para primeira projeção da argamassa nas placas, isto é, a aplicação do chapisco com espessura de aproximadamente de $0,5 \mathrm{~cm}$. Logo, para preparar a argamassa, foi adotado um traço de 1:3 (01 parte de cimento para 03 partes de areia média). Após três dias, os compósitos foram revestidos com argamassa de traço de 3:2:1 (areia: areóla: cimento). Essa etapa final da composição dos compósitos pode ser visualizada na (Figura 1). 
Figura 1 - Tijolo compósito tipo sanduiche etapa final.

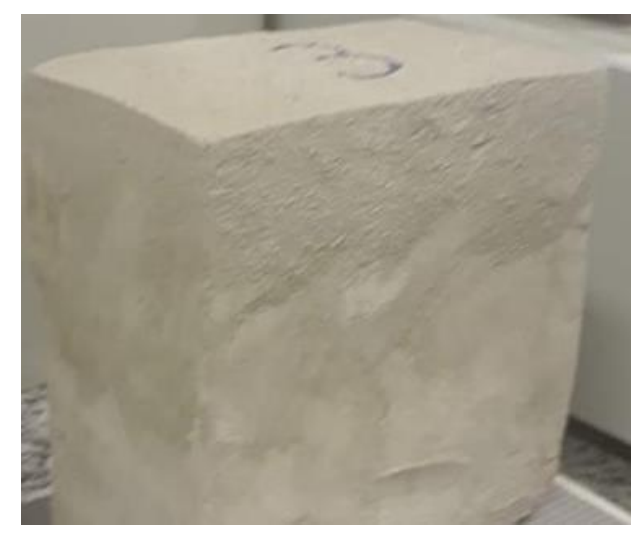

Fonte: Acervo do autor, 2019.

Posteriormente à finalização da composição dos compósitos, estes só foram utilizados para ensaios, a partir de um processo de cura de 28 dias.

\subsection{Metodologia dos ensaios}

Para a realização dos ensaios, foram usados 18 corpos de provas, executados nas etapas mencionadas anteriormente. As divisões dos CPs foram as seguintes:

a) 3 (três) CPs normais;

b) 3 (três) CPs para o ensaio de Absorção;

c) 3 (três) CPs para o processo de aplicação da temperatura de $50^{\circ} \mathrm{C}$ no período de $2 \mathrm{~h}$;

d) 3 (três) CPs para o processo de aplicação da temperatura de $50^{\circ} \mathrm{C}$ no período de $4 \mathrm{~h}$;

e) 3 (três) CPs para o processo de aplicação da temperatura de $50^{\circ} \mathrm{C}$ no período de $6 \mathrm{~h}$;

f) 1 (um) CP para o processo de aplicação da temperatura de $180^{\circ} \mathrm{C}$ no período de $24 \mathrm{~h}$;

g) 1 (um) CP para o ensaio de envelhecimento natural;

h) 1 (um) CP para o ensaio de salinidade.

\subsubsection{Etapas dos ensaios}

Os compósitos passaram pelo processo de cura, foram levados ao laboratório e numerados. Posteriormente, aconteceram às pesagens deles.

No ensaio de absorção, após a pesagem, os compósitos foram imersos em água, por um período de 24 horas, com posterior eliminação da água na superfície, pesagem e avaliação da massa saturada. Logo após esse tempo, eles foram pesados, com a finalidade de obter a massa final.

Para realizar um comparativo do material estudado, também foram imersos em água três tijolos cerâmicos (modelo apresentado). Após suas pesagens, estes apresentaram, aproximadamente, as dimensões dos compósitos. A absorção de água foi determinada de acordo com o que preconiza a ABNT NBR 15270-3 
(ASSOCIAÇÃO..., 2005) para blocos cerâmicos

Para calcular a porcentagem de absorção, foi utilizada a equação a seguir:

- $\%$ Abs $=(M 2-M 1 / M 1) \times 100$

Onde:

- $\mathrm{M} 1=\mathrm{m} 1$ é a massa do corpo de prova seco, expressa em gramas (g);

- $\mathrm{M} 2=$ é a massa do corpo de prova saturado, expressa em gramas (g).

Os corpos de provas foram submetidos ao efeito da temperatura. Logo, os corpos de provas foram submetidos à variadas temperaturas, tendo a primeira etapa se dado com a submissão de um deles a uma temperatura de $180^{\circ} \mathrm{C}$, no tempo de $24 \mathrm{~h}$, em uma estufa. Os outros corpos de prova foram colocados na estufa, sendo um por vez, até atingirem a temperatura pretendida de $50^{\circ} \mathrm{C}$ que, uma vez alcançada, proporcionou que 3 CPS fossem mantidos no equipamento durante 2 horas, 3 CPS durante 4 horas e outros 3 CPS mantidos durante 6 horas, sendo esses retirados do forno e ensaiados quando já se encontravam à temperatura ambiente.

O ensaio de resistência à compressão foi realizado no Instituto Nacional de Tecnologia (INT), sendo utilizada Máquina Universal de Ensaios da Marca Instron (modelo 3382), equipada com célula de carga Instron.

Posteriormente ao ensaio de compressão realizado com limite máximo de $100 \mathrm{kN}$, e força máxima de deformação de $84 \%$ a $86 \%$, foram medidas as espessuras dos CPs, pois estes passaram por uma considerável deformação (Figura 2).

Figura 2 - Deformação do compósito (A) é medidas das espessuras (B).

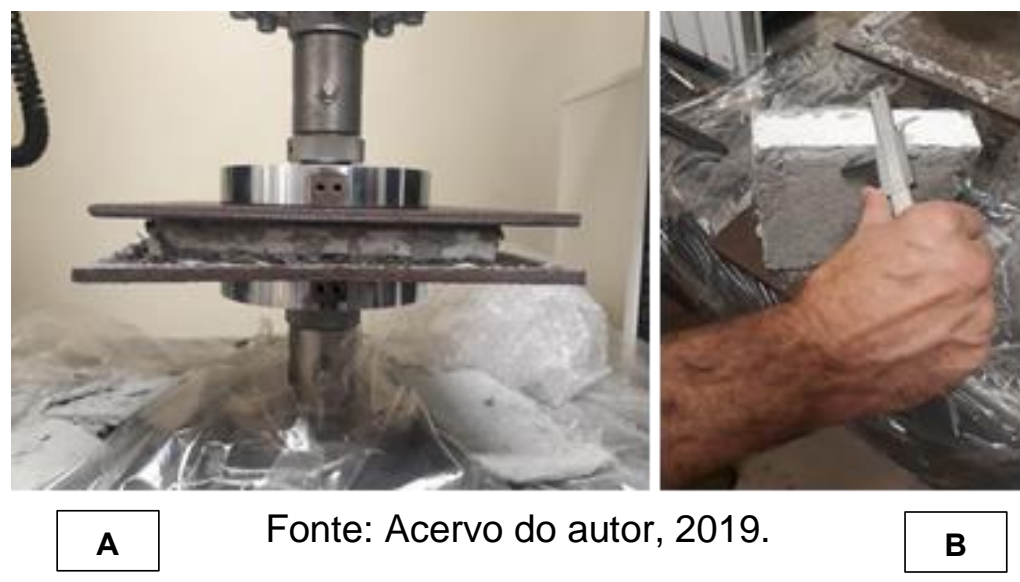

\section{RESULTADOS E DISCUSSÃO}

O CP submetido a estufa em $180^{\circ} \mathrm{C}$, apresentou uma perda de massa específica de aproximadamente $30,76 \%$, dado esse comprovado no ensaio de compressão. Por meio desse ensaio, pode-se perceber, visualmente, que o EPS e o aço utilizados foram fundidos devido à alta temperatura. 
Observa-se, também, perda de massa quando os CPs foram submetidos a uma aplicação da temperatura de $50{ }^{\circ} \mathrm{C}$, sendo em média $0,50 \%$ para os quais permaneceram $2 \mathrm{~h}$ na estufa, e uma média de média de 2,54\% para os que ficaram por $4 \mathrm{~h}$. Os CPs que permaneceram em período de $6 \mathrm{~h}$, por sua vez, tiveram perdas variáveis, tendo como média $5,52 \%$.

Através do ensaio de absorção de água, pode-se realizar um comparativo entre os compósitos e os tijolos cerâmicos. Logo, diante dos resultados, percebe-se que absorção de água dos tijolos está muito superior ao que estabelece a NBR 15310 (2005), chegando a $80 \%$. Já os compósitos apresentaram cerca de $11,11 \%$, dado muito inferior ao dos tijolos. Sendo assim, nota-se que os compósitos estudados absorvem um teor baixo de água e, desse modo, esse aspecto garante a manutenção das características térmicas e mecânicas deles.

Através do ensaio de compressão, foram obtidos os resultados conforme $s$ quadros 1 e 2 :

Quadro 1 - Força máxima.

\begin{tabular}{|r|l|l|c|c|c|}
\hline & CP ID & Altura $(\mathbf{m m})$ & $\begin{array}{c}\text { Espessura } \\
(\mathbf{m m})\end{array}$ & Largura (mm) & $\begin{array}{c}\text { Forca Máxima } \\
(\mathbf{N})\end{array}$ \\
\hline $\mathbf{1}$ & CP-15 & 150.00 & 230.00 & 230.00 & 7075 \\
\hline $\mathbf{2}$ & CP-06 & 150.00 & 230.00 & 230.00 & 88677 \\
\hline $\mathbf{3}$ & CP-13 & 150.00 & 230.00 & 230.00 & 75844 \\
\hline $\mathbf{4}$ & CP-07 & 150.00 & 230.00 & 230.00 & 85961 \\
\hline $\mathbf{5}$ & CP-11 & 150.00 & 230.00 & 230.00 & 43863 \\
\hline $\mathbf{6}$ & CP-10 & 150.00 & 230.00 & 230.00 & 60311 \\
\hline $\mathbf{7}$ & CP-09 & 150.00 & 230.00 & 230.00 & 105005 \\
\hline $\mathbf{8}$ & CP-16 & 150.00 & 230.00 & 230.00 & 95953 \\
\hline $\mathbf{9}$ & CP-19 & 150.00 & 230.00 & 230.00 & 92551 \\
\hline $\mathbf{1 0}$ & CP-12 & 150.00 & 230.00 & 230.00 & 83846 \\
\hline $\mathbf{1 1}$ & CP-18 & 150.00 & 230.00 & 230.00 & 71422 \\
\hline $\mathbf{1 2}$ & CP-17 & 150.00 & 230.00 & 230.00 & 105003 \\
\hline $\mathbf{1 3}$ & CP-14 & 150.00 & 230.00 & 230.00 & 82900 \\
\hline $\mathbf{1 4}$ & CP-20 & 150.00 & 230.00 & 230.00 & 98837 \\
\hline $\mathbf{1 5}$ & CP-08 & 150.00 & 230.00 & 230.00 & 76819 \\
\hline
\end{tabular}

Fonte: Acervo do autor, 2019. 
Quadro 2 - Tensão e deformação.

\begin{tabular}{|c|c|c|c|}
\hline & $\begin{array}{c}\text { Tensão na } \\
\text { Forca Máxima } \\
(\mathbf{M P a})\end{array}$ & $\begin{array}{c}\text { Deformação na } \\
\text { Forca Máxima } \\
\mathbf{( \% )}\end{array}$ & $\begin{array}{c}\text { Taxa de } \\
\text { Deslocamento } \\
(\mathbf{m m} / \mathbf{m i n})\end{array}$ \\
\hline $\mathbf{1}$ & 0 & 37 & 10 \\
\hline $\mathbf{2}$ & 2 & 85 & 10 \\
\hline $\mathbf{3}$ & 1 & 85 & 10 \\
\hline $\mathbf{4}$ & 2 & 86 & 10 \\
\hline $\mathbf{5}$ & 1 & 86 & 10 \\
\hline $\mathbf{6}$ & 1 & 86 & 10 \\
\hline $\mathbf{7}$ & 2 & 84 & 10 \\
\hline $\mathbf{8}$ & 2 & 84 & 10 \\
\hline $\mathbf{9}$ & 2 & 85 & 10 \\
\hline $\mathbf{1 0}$ & 2 & 86 & 10 \\
\hline $\mathbf{1 1}$ & 1 & 86 & 10 \\
\hline $\mathbf{1 2}$ & 2 & 86 & 10 \\
\hline $\mathbf{1 3}$ & 2 & 86 & 10 \\
\hline $\mathbf{1 4}$ & 2 & 86 & 10 \\
\hline $\mathbf{1 5}$ & 1 & 86 & 10 \\
\hline
\end{tabular}

Fonte: Acervo do autor, 2019.

O parâmetro adotado para avaliar a forma máxima atuante a deformação na força máxima de $85 \%$; logo, verifica-se que os CP 11 e CP 10 que foram imersos apresentaram uma resistência menor à compressão. Por outro lado, apesar dos CPS submetidos à temperatura de $50^{\circ} \mathrm{C}$ terem perdido massa específica, os resultados demostram que não houve perda da resistência à compressão, e a deformação manteve-se, quando comparado aos resultados dos outros CPs que não passaram por processo. Porém, observou-se que houve uma redução na resistência à compressão dos corpos de prova aquecidos a $180^{\circ} \mathrm{C}$, submetidos às $24 \mathrm{~h}$ horas na estufa.

Analisando a deformação de 10\% estabelecida pela NBR 8082 (ASSOCIAÇÃO..., 2016), o CP que teve uma resistência menor à compressão de de $96.41 \mathrm{kN} / \mathrm{m}^{2}$ ou 9,64 tf $\mathrm{m}^{2}$, logo, pode-se concluir que mesmo o compósito que apresentou menor resistência, suportou uma carga maior que $8,1 \mathrm{tf} / \mathrm{m}^{2}$ do EPS, apresentada pela NBR 8082 (ASSOCIAÇÃO..., 2016). Nesse contexto, pode-se deduzir que o aço e a argamassa oferecem uma resistência maior ao material.

Nesse cenário, pode se dizer que os compósitos estudados são capazes de suportar as cargas estabelecidas pela NBR 6120 (ASSOCIAÇÃO..., 1980), norma essa que fixa as condições exigíveis para determinação dos valores das cargas que devem ser consideradas no projeto de estrutura de edificações. Eles podem ser adotados até mesmo em casas de máquinas, para as quais a norma sugere o valor mínimo de 7,5 $\mathrm{kN} / \mathrm{m} 2$.

Diante desse resultado, observa-se que o compósito que foi imerso não atenderia, pois a sua resistência a compressão é de $96.41 \mathrm{kN} / \mathrm{m}^{2}$. Porém, os compósitos que apresentaram uma resistência superior a $92,55 \mathrm{kN}$, com uma deformação de $86 \%$, poderiam ser adotados, pois apresentaram uma resistência de $203,43 \mathrm{kN} / \mathrm{m}^{2}$. Torna-se relevante ressaltar, ainda, que os cálculos são específicos para os exemplos 
apresentados.

Analisa-se o compósito como alvenaria à compressão simples, de acordo a NBR 10837 (ASSOCIAÇÃO...,2000), observa-se que o compósito que foi imerso não atenderia, pois a sua resistência a compressão é de $96.41 \mathrm{kN} / \mathrm{m}^{2}$. Porém, os compósitos que apresentaram uma resistência superior a $92,55 \mathrm{kN}$, com uma deformação de $86 \%$, poderiam ser adotados, pois apresentaram uma resistência de $203,43 \mathrm{kN} / \mathrm{m}^{2}$. Torna-se relevante ressaltar, ainda, que os cálculos são específicos para os exemplos apresentados.

\section{CONCLUSÕES}

Para os ensaios realizados, foram utilizados 18 (dezoito) CPS e duas amostras de tijolos. Nesse contexto, foi observado que o compósito não suporta uma aplicação da temperatura de $180^{\circ} \mathrm{C}$ no período de $24 \mathrm{~h}$, pois o EPS e o aço utilizados são fundidos devido à alta temperatura. Porém, tratando-se dos que foram submetidos a uma temperatura de $50^{\circ} \mathrm{C}$ no período de $2 \mathrm{~h}$, $4 \mathrm{~h}$ e $6 \mathrm{~h}$, foi observado que houve perda massa específica, porém, esse fenômeno não comprometeu a perda da resistência à compressão, e a deformação manteve-se, quando se comparado aos resultados dos outros CPS que não passaram por esse processo.

Os CPS que foram imersos apresentaram uma absorção de água menor quando comparados aos tijolos. Logo, por meio dos resultados, nota-se que os compósitos estudados absorvem um teor baixo de água.

Pode-se, também, deduzir que o aço e a argamassa oferecem uma resistência maior ao material, pois o compósito que apresentou menor resistência, suportou uma carga maior que $8,1 \mathrm{tf} / \mathrm{m}^{2}$ do EPS, a qual é indicada pela NBR 8082 (2016).

Nesse cenário, pode se dizer que os compósitos estudados são capazes de suportar as cargas quando estão distribuídas por $\mathrm{m}^{2}$. Porém, quando analisado o compósito como alvenaria à compressão simples (parede), só atenderiam os que apresentaram uma resistência superior a $92,55 \mathrm{kN}$ e uma deformação de $86 \%$. Apesar dos dados obtidos deve-se realizar novos estudos com EPS que apresentam uma densidade maior do que os usados nos compósitos.

\section{REFERÊNCIAS}

ASSOCIAÇÃO BRASILEIRA DE NORMAS TÉCNICAS (ABNT). NBR 6120: cargas para o cálculo de estruturas de edificações - Métodos de ensaio. Rio de Janeiro, 1980.

ASSOCIAÇÃO BRASILEIRA DE NORMAS TÉCNICAS (ABNT). NBR 10837: cálculo de alvenaria estrutural de blocos vazados de concreto - Procedimentos. Rio de Janeiro, 2000. 
ASSOCIAÇÃO BRASILEIRA DE NORMAS TÉCNICAS (ABNT). NBR 15270-3: componentes cerâmicos parte 3: Blocos cerâmicos para alvenaria estrutural e de vedação - Métodos de ensaio. Rio de Janeiro, 2005.

ASSOCIAÇÃO BRASILEIRA DE NORMAS TÉCNICAS (ABNT). NBR 8082: espuma rígida de poliuretano para fins de isolação térmica - determinação da resistência à compressão. Rio de Janeiro, 2016.

ASSOCIAÇÃO BRASILEIRA DO POLIESTIRENO EXPANDIDO. O que é EPS. 2010. Disponível em: http://www.abrapex.com.br/010queeEPS.html. Acesso em: 05 mar. 2019.

CALDEIRA, Débora; SPOSTO, Rosa; CALDAS, Lucas. Avaliação do ciclo de vida energético de telha termoacústica com poliestireno expandido e telha cerâmica tipo plana em edificação unifamiliar. In: Encontro Nacional de Tecnologia do Ambiente Construído,16., 2016. Anais..., Santa Maria, 2016.

JOHN, V. M.; SATO, N. M. N. Durabilidade de componentes da construção. In: SATTLER, M. A.; PEREIRA, F. O. R. (Org.). Construção e Meio Ambiente. Porto Alegre: ANTAC, 2006. p. 21-57.

JOHN, V. M.; SJÖSTRÖM, C.; AGOPYAN, V. Durability in the Built Environment and Sustainability in Developing Countries. In: INT. CONF. ON DURABILITY OF BUILDING MATERIALS AND COMPONENTS, 9 th, 2002.Proceedings..., Brisbane. Melbourne: CSIRO, 2002. p. 11-1-11-7.

LOPES, Gil Alves. Avaliação do ciclo de vida de dois materiais de isolamento utilizados na construção civil: o poliestireno expandido e o aglomerado de cortiça expandida. 107f. 2011. Dissertação (Mestrado em Engenharia do Ambiente) Universidade do Porto, Porto, 2011.

MATEUS, Ricardo; BRAGANÇA, Luís. Avaliação da sustentabilidade da construção: desenvolvimento de uma metodologia para a avaliação a sustentabilidade de soluções construtivas. In: CONGRESSO SOBRE CONSTRUÇÃO SUSTENTÁVEL, 1., 2004. Anais..., Leça da Palmeira, 2004.

SANTOS, Maria Betania Gama et al. Sustentabilidade na construção civil: utilização do resíduo de poliestireno expandido como material não convencional. In: ENCONTRO NACIONAL DE ENGENHARIA DE PRODUÇÃO, 29., 2009. Anais..., Salvador, 2009.

SANTOS, Reginaldo Dias dos. Estudo Térmico e de Materiais de um Compósito à Base de Gesso e EPS para Construção de Casas Populares. 2008. Dissertação de Mestrado. Universidade Federal do Rio Grande do Norte, 2019.

SANTOS, Tamires Polydoro Saldanha. Reciclagem de poliestireno expandido: compósito com fibras de algodão para a confecção de placas de circuito impresso. $55 f$. 2013. Trabalho de conclusão de curso (Bacharelado Engenharia de Materiais) Universidade Estadual Paulista, Guaratinguetá, 2013.

STOCCO, Wagner; RODRIGUES, David; CASTRO, A. P. A. S. Concreto leve com uso de EPS. Cobenge, v. 32, n. 1, p. 47-55, 2009. 